Fetal Diagnosis

and Therapy
Fetal Diagn Ther 2008;24:230-236

DOI: 10.1159/000151344
Received: May 21, 2007

Accepted after revision: July 12, 2007

Published online: August 28, 2008

\title{
Longitudinal Determination of Serum Placental Protein 13 during Development of Preeclampsia
}

\author{
Berthold Huppertz ${ }^{a} \quad$ Marei Sammar ${ }^{b} \quad$ Ilana Chefetz ${ }^{b}$ \\ Peruka Neumaier-Wagner ${ }^{c}$ Clemens Bartz ${ }^{d}$ Hamutal Meirib \\ a Institute of Cell Biology, Histology and Embryology, Centre of Molecular Medicine, Medical University of Graz,

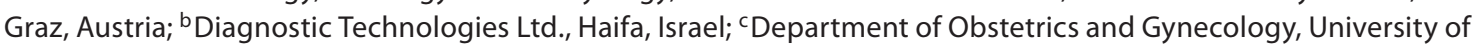 \\ Munich, Munich, and d Department of Obstetrics and Gynecology, University RWTH Aachen, Aachen, Germany
}

\section{Key Words}

Preeclampsia $\cdot$ Placental protein $13 \cdot$ Placenta $\cdot$ Maternal serum · Prediction • Marker

\begin{abstract}
Objective: To determine maternal serum placental protein 13 (PP13) in normal pregnancy and preeclampsia. Methods: A prospective, longitudinal study with 41 normal pregnant women, 18 cases with preterm delivery or cervix insufficiency and 4 with developing late-onset preeclampsia. Six hundred and sixty-six maternal blood samples were obtained every 2-4 weeks starting at 5-8 weeks gestation (10-12 samples/patient) and tested for serum PP13 by ELISA. Results: In normal pregnant women delivering at term, median maternal serum PP13 levels were growing from 166 to $202 \mathrm{pg} / \mathrm{ml}$ and $382 \mathrm{pg} / \mathrm{ml}$ in the first, second and third trimester, respectively. Preeclamptic women had significantly reduced PP13 levels in the first trimester (multiples of median of 0.14 at 7-8 weeks; $p=0.005$ compared to normal). PP13 in the third trimester was significantly higher compared to normal at 35-36 weeks with PP13 multiples of median of 1.79. Conclusion: This preliminary study indicates that low levels of PP13 in early pregnancy identify at-risk pregnancies, whereas high levels precede the syndrome in late pregnancy and suggest syncytiotrophoblast necrosis.
\end{abstract}

Copyright $\odot 2008$ S. Karger AG, Basel

\section{Introduction}

Preeclampsia is a common syndrome and remains a major cause of maternal and fetal morbidity and mortality $[1,2]$. Most women develop the late-onset syndrome with normal fetal growth, whereas a smaller subset develops the early-onset syndrome usually accompanied by intrauterine growth restriction (IUGR) [3, 4]. Both types of the syndrome are characterized by systemic inflammation that resolves following delivery. Since trophoblast fragments and proteins, microvillous membranes and cell-free fetal DNA are all detectable in higher amounts in maternal blood of preeclamptic women, the placenta is the likely source of this inflammatory disorder [3].

The prevention of preeclampsia remains a considerable challenge in obstetrics. Neither low-dose aspirin [5] nor antioxidant vitamins $[6,7]$ were shown to be helpful in preventing any type (early- or late-onset) of preeclampsia. One reason why preventive strategies for this syndrome have proven so disappointing at present is that the proposed interventions have commenced in the mid to late second trimester, when the underlying placental disorder triggering the syndrome may already be established. Thus, novel biomarkers of the syndrome expressed in the first trimester to predict pregnancies at risk may permit new interventions to try and prevent this placentally mediated syndrome.

\section{KARGER}

Fax +41613061234 E-Mail karger@karger.ch www.karger.com
Prof. Berthold Huppertz

Institute of Cell Biology, Histology and Embryology, Centre of Molecular Medicine Medical University of Graz, Harrachgasse 21/7, AT-8010 Graz (Austria)

Tel. +43 316380 7604, Fax +433163809625

E-Mail berthold.huppertz@meduni-graz.at 
Recently, the concentrations of placental protein 13 (PP13) [8] were shown to be altered in maternal blood in pregnancy disorders such as preeclampsia [9]. More recent studies have shown that the serum levels of PP13 are significantly reduced at 11 to $13 \pm 6$ weeks in cases developing early- as well as late-onset preeclampsia [10, 11]. On the background of these data, we analyzed cases developing late-onset preeclampsia longitudinally throughout their pregnancies. PP13 is expressed in the placental syncytiotrophoblast, and its levels in maternal blood may reflect turnover and integrity of this layer. Our data suggest a biphasic alteration in maternal expression of PP13 during the evolution of pregnancies that ultimately develop preeclampsia.

\section{Materials and Methods}

\section{Study Design and Population}

The prospective study involved the Women's Hospital at the University of Zurich, Switzerland and a collective of doctors in private practice at Nordhorn, Germany. The study was approved by the ethical committees of both centers and women were enrolled after providing informed consent. Patients were followed from the first positive $\beta$-hCG test at enrolment throughout antenatal visits during pregnancy until delivery, and outcome was documented in the early postpartum period. Five-milliliter aliquots of blood were provided every 2-4 weeks from enrolment until 5-15 weeks after delivery. Serum was stored frozen at $-70^{\circ} \mathrm{C}$ until tested. Demography, maternal medical history, results of blood pressure measurements, urinalyses and laboratory tests were collected and recorded within an electronic database, as were the later pregnancy outcomes (table 1).

Women aged 18-45 years with a singleton gestation who delivered after 20 weeks were included in the current study. The total number of patients enrolled was 92 , of which 19 were excluded due to missed abortions or incompliance (no blood or loss of follow-up). Ten patients with multiple admissions of tocolytic agents were excluded due to tocolytic effects on PP13 serum levels discovered post factum, which will be reported elsewhere [Huppertz, pers. commun.]. Accordingly, the current report describes the results obtained from 63 patients, 4 of them preeclamptic and 59 unaffected. The 4 patients who developed preeclampsia correspond to $4.5 \%$, as expected from previous epidemiological reports, and the 59 unaffected patients are subdivided into 41 with normal outcome, 8 who delivered preterm ( $<37$ weeks) and 10 with short cervix (length $<25 \mathrm{~mm}$, according to the American College of Obstetricians and Gynecologists) [12]. None of the preterm cases was complicated by preterm preeclampsia.

Preeclampsia was defined according to the classification of the International Society for the Study of Hypertension in Pregnancy $[13,14]$, namely hypertension that developed after 20 weeks in a previously normotensive woman and was coupled to proteinuria exceeding $300 \mathrm{mg}$ in 24 -hour collections or $2+$ in dipstick testing. A total of 664 blood samples (10-12 of each patient) were obtained from the 59 unaffected women and the 4 preeclamptic patients.

\section{PP13 Testing}

Maternal serum concentration of PP13 was measured blinded to pregnancy outcome using a solid-phase sandwich ELISA with a pair of PP13-specific monoclonal antibodies, marked with amplified biotin-extravidin-horseradish-peroxidase complex and developed with tetramethylbenzidene substrate, as previously described $[9,10]$. Optical density was measured at $450 \mathrm{~nm}$ against a 650 -nm background and translated to a quantitative amount by using a calibration curve made of recombinant PP13 standards $(0-500 \mathrm{pg} / \mathrm{ml})$. The assay's operating range is $5-400 \mathrm{pg} / \mathrm{ml}$, and samples with lower readings were assigned the value $5 \mathrm{pg} / \mathrm{ml}$. The intra- and interassay coefficients of variation for this study were 5.3 and $9.5 \%$, respectively, while the recovery coefficient of variation was $11.5 \%$.

\section{Statistical Analysis}

Baseline and delivery characteristics were compared between cases and controls using the Fisher exact test for categorical variables and independent $t$ tests for continuous variables.

PP13 levels were not normally distributed; therefore, we compared PP13 levels across the 2 outcome groups using the Wilcoxon rank sum test. PP13 levels increased with gestational age. To take this effect into account, PP13 levels were converted to multiples of the normal median (MoM) for the controls at the same gestational age, based on a weighted (by number of cases) regression of the observed median at each completed week. We examined other major confounders: PP13 did not change with body mass index $(p=0.168)$, age $(p=0.737)$, parity $(p=0.147)$ and ethnicity $(\mathrm{p}=0.606)$. Thus, the calculation of MoM was not further adjusted to these confounders.

\section{Immunohistochemistry}

Term placentas from normal $(\mathrm{n}=5)$ and late-onset preeclampsia $(n=5)$ cases were obtained from the Women's Hospital of Aachen University, Germany and the Wolfson Medical Centre in Holon, Israel at the time of this study after obtaining informed consent and the placentas were then used for immunohistochemistry. These 10 cases were not related to the cases tested for serum PP13.

A tissue block of each placenta was fixed in $4 \%$ formaldehyde, dehydrated in a graded series of ethanol and embedded in paraffin. Five-micrometer sections were cut, mounted on glass slides and deparaffinized. Immunohistochemical staining was performed with monoclonal primary antibodies against PP13 (monoclonal antibodies 27-2-3 and 215-28-3) at a dilution of 1:10. Positive staining appeared red after using a standardized staining sequence based on the horseradish peroxidase-linked streptavidin-biotin technique for the detection of a biotinylated secondary antibody and AEC detection reagent. Control reactions excluded the use of primary antibodies or made use of a negative control antibody (DAK-GO1; Dako) and were always negative.

\section{Results}

\section{Demography and Delivery Characteristics}

Table 1 displays the baseline and delivery characteristics in relation to pregnancy outcome. Women were of 
Table 1. Demographic characteristics of women in the 3 groups (control, unaffected and late-onset preeclampsia)

\begin{tabular}{lccc}
\hline & $\begin{array}{c}\text { Normal } \\
(\mathrm{n}=41)\end{array}$ & $\begin{array}{c}\text { Unaffected } \\
(\mathrm{n}=59)\end{array}$ & $\begin{array}{c}\mathrm{PE} \\
(\mathrm{n}=4)\end{array}$ \\
\hline Maternal age at enrolment, years & $31 \pm 5$ & $33 \pm 7$ & $30 \pm 4$ \\
GA at enrolment, weeks & $7.8 \pm 2.2$ & $6.8 \pm 2.7$ & $9.1 \pm 2.1$ \\
Caucasians, \% & 60 & 58 & 50 \\
Nulliparous, \% & 41 & 44 & 50 \\
More than 1 pregnancy loss/abortion, $\%$ & 32 & 32 & 50 \\
Smoking, \% & 24 & 27 & 0 \\
Blood pressure at enrolment, mm Hg & $\leq 110 / 73$ & $\leq 107 / 81$ & $\leq 140 / 90$ \\
Elevated blood pressure at GA, weeks & - & - & $35.0 \pm 3.0$ \\
Proteinuria (dipstick) & traces & traces & $2+(2+$ to $4+)$ \\
GA at delivery, weeks & $39.9 \pm 1.1$ & $38.2 \pm 1.7$ & $38.5 \pm 1.1$ \\
Birth weight, g & $3,446 \pm 417$ & $3,017 \pm 547$ & $3,300 \pm 728$ \\
Vaginal delivery, \% & 80 & 75 & 100 \\
\hline
\end{tabular}

Values are medians $\pm 95 \% \mathrm{CI}$.

$\mathrm{PE}=$ Late-onset preeclampsia; $\mathrm{GA}=$ gestational age . similar age at enrolment $(31 \pm 5,33 \pm 7$ and $30 \pm 4$ years for normal control, unaffected and preeclamptic women, respectively) and were enrolled at a similar gestational age $(7.8 \pm 2.2,6.8 \pm 2.7$ and $9.1 \pm 2.1$ weeks, respectively). About $60 \%$ of the enrollees were Caucasian in either group. The preeclamptic and normal outcome group women delivered at term; delivery of the unaffected group was lower due to the patients who delivered preterm (35.2 \pm 1.4 weeks). All preeclamptic patients delivered by vaginal delivery ( $80 \%$ in the normal outcome group and $70 \%$ in the unaffected group in which $6 / 8$ preterm cases and $4 / 10$ short cervix cases delivered by caesarean section). Baby birth weight was similar in the control group $(3,446$ $\pm 417 \mathrm{~g})$ compared to the preeclamptic group $(3,300 \pm$ $728 \mathrm{~g})$, but lower in the unaffected group $(3,017 \pm 547 \mathrm{~g})$ due to the preterm delivery cases $(2,100 \pm 330 \mathrm{~g})$.

At enrolment, all patients had normal blood pressure and none or traces of proteinuria. At $35 \pm 3$ weeks, both systolic and diastolic blood pressure of preeclamptic patients were elevated above 140/90 with proteinuria of $2+$ ( $95 \%$ CI: $2+$ to $4+$ ).

\section{PP13 Blood Levels}

Following each individual patient with normal outcome, it was discovered that their values did not change much during the first trimester, although fluctuations around the median values indicated a tendency towards higher values near the time that pregnancy was established by the $\beta$-hCG test and at the end of the first trimester (fig. 1a, solid line). For each individual patient, the values were on average $25 \%$ higher in the second tri- mester and approximately double this amount towards term at around 35-36 weeks. At 8-12 weeks after delivery (corresponding to 41-45 and 46-50 weeks from the last menstrual period), PP13 disappeared from the maternal serum (fig. 1a, solid line), thus enabling us to use this as a baseline to correct the values of each individual woman. This approach improved the accuracy of the analysis.

To obtain the cross-section of the 41 normal patients (430 blood samples), median PP13 values with 95\% CI were calculated (table 2). Median first trimester PP13 level adjusted to gestational week after weighted (by number of patients) regression was $166 \mathrm{pg} / \mathrm{ml}$ (range 126-202). The median second trimester level adjusted to gestational age was $202 \mathrm{pg} / \mathrm{ml}$ (range 171-233), reflecting an average increase of $25 \%$ between the first and the second trimester. Values for individuals increased $50-100 \%$ from the first trimester to the maximum value reached during the third trimester. PP13 disappeared from maternal blood after delivery, indicating that serum PP13 is directly related to pregnancy and the presence of the placenta (table 2; fig. 1a, solid line).

Comparison of the normal group to the total unaffected group [including cases that subsequently delivered preterm or those with a relatively shorter cervix (fig. 1a, triangles)] indicated that the normal and unaffected women almost overlap and the differences were not significant throughout pregnancy (table 2).

In contrast to the above, the level of PP13 in preeclampsia cases (51 blood samples collected from 4 patients) started lower than normal (fig. la, dashed line). 
Fig. 1. a PP13 in patients' serum throughout pregnancy. Course of PP13 release into maternal blood in normal pregnant women ( $\mathrm{n}=41$; solid line), in unaffected women ( $\mathrm{n}=59$; triangles, no line) and in patients who developed late-onset preeclampsia at term $(\mathrm{n}=4$; dashed line). Each woman gave 10-12 blood samples after enrolment. Data are represented as medians with 95\% CI of intervals of 2 weeks. Weeks are presented after last menstrual period. Accordingly, weeks 41-55 represent 1-15 weeks after delivery. b Comparative view on the time course of PP13 release (solid line; $\mathrm{R}^{2}=0.9677$ ) relative to data published elsewhere [17] showing growth of villous surface (dotted line; $\mathrm{R}^{2}=1.0$ ) and growth of trophoblast volume (dashed line; $\mathrm{R}^{2}=$ 1.0) throughout gestation. For PP13, the $y-$ axis shows picogram of PP13 per milliliter of maternal peripheral serum, for villous surface, the $y$-axis shows square centimeters (original values $\times 3 / 1,000$ ) and for trophoblast volume, the $y$-axis shows cubic centimeters (original values $\times 5$ ). The values for villous surface and trophoblast volume are taken from Mayhew and Barker [17].

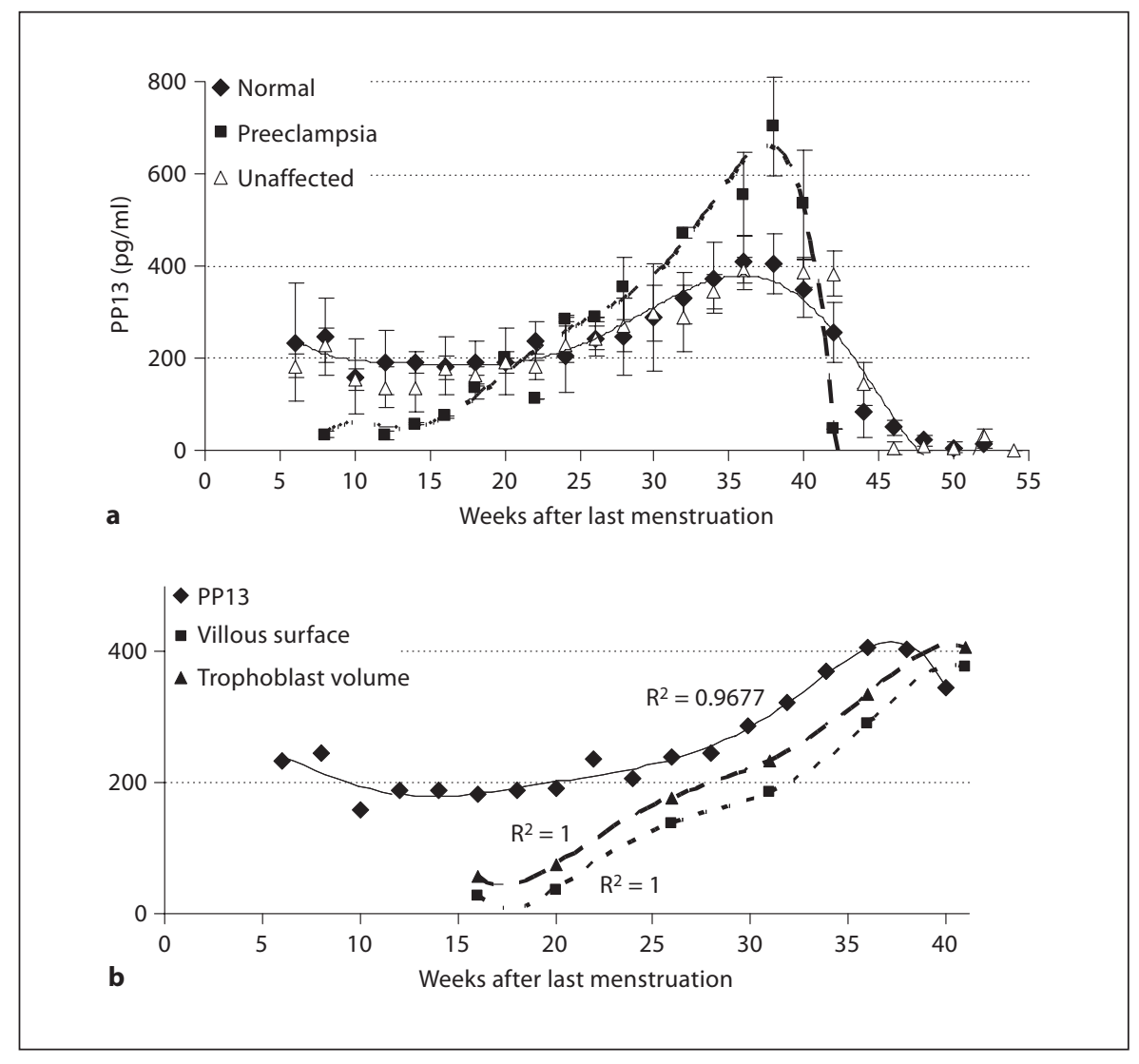

Table 2. PP13 MoM values throughout pregnancy

\begin{tabular}{|c|c|c|c|c|c|c|c|c|c|c|}
\hline \multirow{3}{*}{$\begin{array}{l}\text { Weeks after } \\
\text { LMP }\end{array}$} & \multicolumn{2}{|l|}{ Normal $(n=41)$} & \multicolumn{2}{|c|}{ Unaffected $(\mathrm{n}=59)$} & \multicolumn{6}{|c|}{ Late-onset preeclampsia } \\
\hline & \multirow{2}{*}{$\begin{array}{l}\text { median } \\
\text { PP13 }\end{array}$} & \multirow[t]{2}{*}{ MoM } & \multirow{2}{*}{$\begin{array}{l}\text { median } \\
\text { PP13 }\end{array}$} & \multirow[t]{2}{*}{ MoM } & \multicolumn{4}{|c|}{ PP13 raw data, No. } & \multirow{2}{*}{$\begin{array}{l}\text { median } \\
\text { PP13 }\end{array}$} & \multirow[t]{2}{*}{ MoM } \\
\hline & & & & & 149 & 63 & 117 & 129 & & \\
\hline $5-10$ & $292(205-379)$ & $1.00(0.70-1.30)$ & $180(132-228)^{* *}$ & $0.68(0.50-0.18)^{* *}$ & 29 & 36 & 34 & 50 & $35^{* *}$ & $0.12(0.08-0.16)^{* *}$ \\
\hline $11-15$ & $306(223-389)$ & $1.00(0.73-1.27)$ & $248(198-306)^{*}$ & $0.81(0.75-1.25)^{*}$ & 71 & 16 & 34 & 57 & $46^{* *}$ & $0.15(0.08-0.22)^{*}$ \\
\hline $16-20$ & $313(223-404)$ & $1.00(0.71-1.29)$ & $268(226-306)$ & $0.86(0.71-1.01)$ & 110 & 137 & 156 & 160 & $147^{* *}$ & $0.47(0.39-0.55)^{*}$ \\
\hline $21-25$ & $329(256-412)$ & $1.00(0.78-1.25)$ & $282(200-360)$ & $0.86(0.72-1.02)$ & & 284 & 259 & 265 & $265^{*}$ & $0.80(0.73-0.87)$ \\
\hline $26-30$ & $381(302-460)$ & $1.00(0.84-1.16)$ & $336(376-396)$ & $0.88(0.68-1.22)$ & 435 & 352 & 381 & 288 & 367 & $0.96(0.82-1.10)$ \\
\hline $31-35$ & $420(352-488)$ & $1.00(0.83-1.21)$ & $393(323-353)$ & $0.94(0.85-1.05)$ & 565 & 543 & 535 & 497 & $539^{*}$ & $1.23(1.13-1.33)^{*}$ \\
\hline $36-40$ & $498(424-572)$ & $1.00(0.85-1.15)$ & $460(383-537)$ & $0.92(0.81-1.03)$ & 937 & 850 & 790 & 990 & $894^{* *}$ & $1.79(1.61-1.98)^{* * *}$ \\
\hline $41-45(\mathrm{PD})$ & $305(195-420)$ & $1.00(0.64-1.37)$ & $291(203-379)$ & $0.95(0.78-1.22)$ & 573 & 550 & 480 & 560 & $529^{* *}$ & $0.89(0.63-1.05)$ \\
\hline 46-50 (PD) & $45(11-110)$ & $1.00(0.84-1.16)$ & $57(33-94)$ & $1.26(1.00-1.45)$ & 86 & 134 & 182 & & $134^{* *}$ & $0.65(0.40-0.95)$ \\
\hline 51-55 (PD) & $23(11-35)$ & $1.00(0.47-1.52)$ & $13(0-21.59)$ & $0.59(0.07-1.07)$ & 6 & 15 & 14 & 50 & 15 & $0.65(0.45-0.85)$ \\
\hline
\end{tabular}

Figures in parentheses are 95\% CI. As weeks were counted from the last menstrual period, weeks 41-45, 46-50 and 51-55 after last menstrual period correspond to 1-5, 6-10 and 11-15 weeks after delivery. PD stands for post delivery values. LMP $=$ Last menstrual period. ${ }^{*} \mathrm{p}<0.05 ;{ }^{* *} \mathrm{p}<0.005$. PP13 raw data of $149,63,117$ and 129 reflect values of individual patients who developed preeclampsia, identified by their study code.

Although differences between individuals occurred, all individuals had very low PP13 values during the period of gestational age 5-15 weeks with median values of 35 and $46 \mathrm{pg} / \mathrm{ml}$ for gestational age 5-10 and 11-15 weeks, respectively, with $\mathrm{p}<0.001$ and 0.01 , respectively, compared to normal cases of the corresponding gestational age (table 2; fig. 1a, dashed line). The standard deviation in this group was very small. In fact, for the very early 
Fig. 2. Immunohistochemistry for PP13 in placenta. A normal term placenta (a) and 2 cases of late-onset preeclampsia cases (bd) stained for PP13. Note the more intense staining for PP13 in the preeclamptic cases especially at the apical membrane of the syncytiotrophoblast and additionally in fetal blood vessels (asterisk). d The frame in c has been enlarged to highlight the disturbed surface of the syncytiotrophoblast showing evidence for necrotic release of subcellular fragments only in preeclampsia (arrows). a $\times 700$. b, c $\times 600$. d $\times 800$.
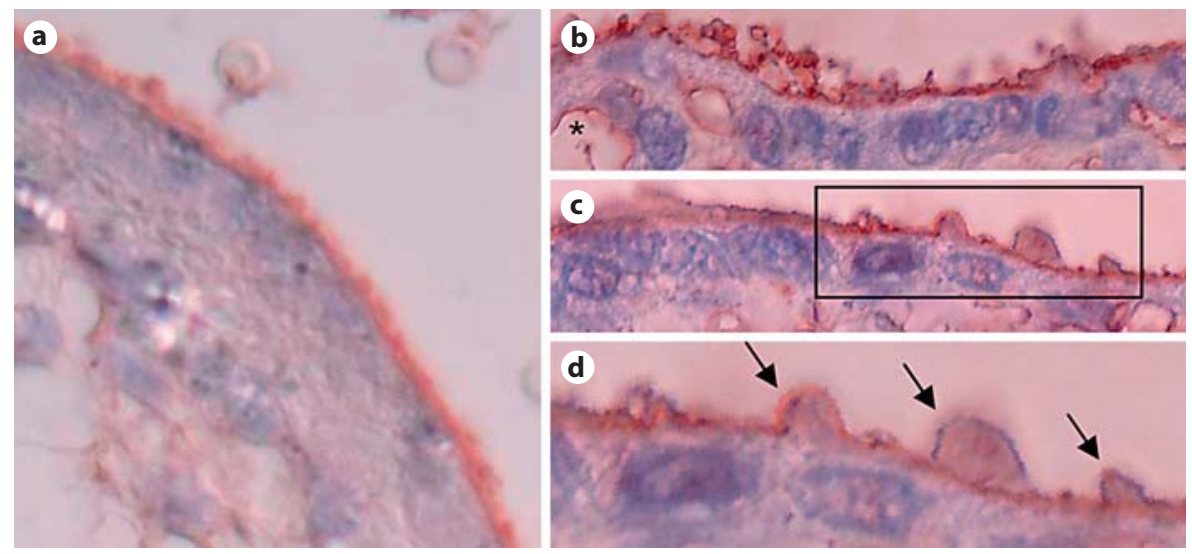

time point of 7 weeks, there was no overlap between the individuals of the 2 groups and all normal cases were higher than preeclamptic patients. During the second trimester, the PP13 values in the preeclampsia cases grew to reach normal levels with medians at gestational age $16-20,21-25$ and $26-30$ weeks growing to 147,265 and $367 \mathrm{pg} / \mathrm{ml}$, respectively $(\mathrm{p}<0.04,0.21$ and 0.75 , respectively; table 2). During the third trimester, PP13 values of the preeclamptic patients overshot above normal with medians at gestational age $31-35$ and 36-40 weeks reaching 539 and $894 \mathrm{pg} / \mathrm{ml}$, respectively ( $\mathrm{p}=0.05$ and 0.001 , respectively; table 2). In the preeclampsia group, PP13 was elevated 7-15 times for each individual patient between the first and the third trimester, whereas average differences for the women of the normal outcome group were 1.4-2 times. After delivery, the values decreased back to normal and then completely disappeared, indicating that serum PP13 is directly related to pregnancy and placenta (table 2; fig. 1a).

\section{PP13 MoM}

Based on medians at each gestational age of the normal outcome group, the corresponding MoM value was set to 1.0. For the late-onset preeclampsia group, the PP13 MoM values were significantly lower than in the normal group at gestational age 5-10 and 11-15 weeks (table 2). During the second trimester, MoM values were growing towards the normal level (table 2). Third-trimester values reached above normal and became significantly different again (table 2) when symptoms of preeclampsia were clinically evident. The change of MoM values for any preeclampsia patient during the entire period reflected initial low MoM values at the period of risk assessment, growing gradually into very high MoM values during the outbreak of clinical symptoms of the pathology. After de- livery, PP13 disappeared from the blood in all cases and controls. Values for the unaffected group were slightly but not significantly lower compared to the normal group (table 2).

\section{Immunohistochemistry}

Immunohistochemical staining for PP13 in human term placentas revealed a clear and nearly exclusive staining of the apical membrane of the syncytiotrophoblast (fig. 2a). With this staining, also the microvillous brush border became visible. In cases from late-onset preeclampsia, staining for PP13 revealed a similar picture (fig. $2 b-d$ ). Here, increased staining of the apical syncytial membrane was found and additional staining of fetal blood vessels was present (fig. 2b, asterisk). In the pathological cases, alterations of the apical membrane became obvious, displaying structures that may lead to the necrotic release of syncytiotrophoblast membrane fragments, known to be increased in preeclampsia [15].

\section{Discussion}

\section{Limitations}

This study was carried out with a relatively small cohort and compares 4 term preeclampsia cases with 59 unaffected cases (41 with normal outcome). While the proportion of cases fits the size of the cohort, the discussion takes into consideration that this is an initial observation that should stimulate the implementation of a larger cohort study. The limited number of cases is slightly misleading, since the longitudinal sampling of 10-12 blood samples from each participant enabled us to gain a quite reliable view on the change over time for 
the normal outcome or the entire unaffected group. Accordingly, each preeclamptic case exhibits a strikingly different temporal change in the PP13 level compared to the unaffected group for any of the time points. The next step is of course associated with repeating the study on a larger scale, as included in the ongoing Pregenesys project.

\section{Correlation between PP13 Concentrations and Normal Development of the Placenta}

Following the concentrations of PP13 in maternal peripheral venous serum, there is a slight reduction around the time of onset of maternal blood flow into the placenta, that is, between weeks 9 and 13 [16]. After the establishment of maternal blood flow through the placen$\mathrm{ta}$, there is a constant increase in PP13 in maternal blood until near term. It peaks at around 36 weeks and then slightly decreases until delivery. Afterwards, it disappears within a week. Figure $1 \mathrm{~b}$ shows the course of PP13 throughout pregnancy in control women (same data as in fig. 1a, solid line) related to the course of other placental parameters that change during pregnancy: trophoblast volume and villous surface (data taken from Mayhew and Barker [17]). It becomes obvious that all 3 courses display the lowest values at the transition from the first to the second trimester followed by a constant increase starting at around midgestation. Since PP13 is expressed in the syncytiotrophoblast outer membrane, it is tempting to speculate that the increase in PP13 nicely reflects the increase in villous surface extension during pregnancy. Any disturbance of the villous surface in terms of surface area or surface integrity will have a direct impact on the amount of PP13 released into the maternal circulation.

\section{Assessing Abnormal Placental Development Already} in the First Trimester of Pregnancy and Allowing to

Test Putative Preventive Therapeutic Interventions

The longitudinal measurements of PP13 throughout pregnancy provide a clear picture of the changes in PP13 release in cases with late-onset preeclampsia. They start with a significantly lower release during the first trimester, cross the normal course at about 20 weeks and peak prior and during the time of clinical symptoms. With these characteristics, the release of PP13 is well suited to distinguish normal pregnancy from cases developing late-onset preeclampsia already during or at the end of the first trimester. This of course will be ameliorated if other factors will be evaluated in parallel as has been done for trisomy 21 [18].

Longitudinal Determination of PP13

during Development of Preeclampsia
Recently, 2 studies have been published concentrating on serum samples from the first trimester $[10,11]$. Both studies showed the same significant reduction of the PP13 levels irrespective of whether the woman developed an early- or a late-onset type of preeclampsia. Furthermore, 2 recent reviews described PP13 as one of the most promising markers to predict preeclampsia $[19,20]$.

The longitudinal first trimester testing of PP13 helps strengthening the prediction of an elevated risk for a later development of preeclampsia and potentially identifying a cutoff for this syndrome. In addition, the repeated testing throughout the second and third trimesters appears to enable differential diagnosis of the anticipated pathology based on the slope of change between consecutive measurements, such as differentiating between earlyand late-onset preeclampsia.

The biphasic changes in the release of PP13 in preeclampsia compared to control cases raises the question as to how these alterations can be explained. The significantly lower values in the first trimester may find their explanation in an abnormal development of the placenta already at this stage of pregnancy. Although there are hypotheses that claim that these alterations should occur very early during placental development, so far no proof for such changes have been presented. The changes in PP13 release may be a first indicator of a dysregulated placental development early in pregnancy resulting in preeclampsia. It has to be noted though, that the reduced amounts of PP13 are true for both groups of preeclampsia, early as well as late onset $[10,11$, this study], while a general explanation for these very low levels early in gestation is missing.

The overshoot of PP13 prior to and during the onset of clinical symptoms of preeclampsia is much easier to explain. It has been shown that trophoblast apoptosis is increased in preeclampsia [21]. Since apoptotic material is cleared in the lungs of pregnant women and will not result in activation of the maternal inflammatory system [22], and since elevated apoptosis has also been described for IUGR [23], increased apoptosis cannot account for the increase in PP13 in preeclampsia. On the other hand, it has been postulated [24] that it is rather an increase in the rates of necrosis and aponecrosis that may account for the increase in proteins and DNA in peripheral blood as well as account for the induction of the inflammatory response. This hypothesis is supported by a recent paper by Goswami et al. [25], who showed that subcellular fragments of the syncytiotrophoblast inside maternal blood are only elevated in preeclampsia but not in IUGR.

Fetal Diagn Ther 2008;24:230-236 


\section{Conclusions}

Testing of PP13 in maternal blood throughout pregnancy may enable us to view a proper placental development. Alterations of placental structure and function may be directly related to changes in the amount of PP13 released into maternal blood. Since such alterations can be visualized already during the course of the first trimester in pregnancy pathologies where a placental cause is expected, PP13 may be used as a tool to assess an abnormal development of the placenta in preeclampsia.

\section{Acknowledgements}

The authors would like to thank Dr. Gundula Hebisch from the Department of Obstetrics and Gynecology, University of $\mathrm{Zu}-$ rich, Switzerland, for providing us with the serum samples, and Uta Zahn from the Department of Anatomy II, University Hospital RWTH Aachen, Germany, for her excellent technical support in performing the immunohistochemical staining. The authors highly appreciated the permission to use the statistical modeling for the normal course of PP13 during pregnancy as developed by Howard Cuckle and Dr. Yossi Tal and the statistical assistance of Ido Kuhnreich.

This study was sponsored in part by a grant from Israel's Chief Scientist (grant No. 31851) to Diagnostic Technologies covering the costs of shipping kits, testing PP13 and advisory statistical support. This study was supported by the European Union FP6 (Pregenesys, grant No. 037244).

\section{References}

1 National Collaborating Centre for Women's and Children's Health: Antenatal Care: Routine Care for the Healthy Pregnant Woman. London, National Institute for Clinical Excellence, 2003.

2 The National Institute for Clinical Excellence, Scottish Executive Health Department; Department of Health, Social Services and Public Safety, Northern Ireland: The Fifth Report of the Confidential Enquiries into Maternal Deaths in the United Kingdom 1997-1999. London, RCOG Press, 2001, pp 76-93.

3 von Dadelszen P, Magee LA, Roberts JM: Subclassification of preeclampsia. Hypertens Pregnancy 2003;22:143-148.

4 Redman CW, Sargent IL: Latest advances in understanding preeclampsia. Science 2005; 308:1592-1594

5 Sibai BM, Caritis SN, Thom E, Klebanoff M, McNellis D, Rocco L, Paul RH, Romero R, Witter F, Rosen M, Depp R, National Institute of Child Health and Human Development Network of Maternal-Fetal Medicine Units: Prevention of preeclampsia with lowdose aspirin in healthy, nulliparous pregnant women. N Engl J Med 1993;329:1213-1218.

6 Poston L, Briley AL, Seed PT, Kelly FJ, Shennan AH, Vitamins in Pre-eclampsia (VIP) Trial Consortium: Vitamin $\mathrm{C}$ and vitamin $\mathrm{E}$ in pregnant women at risk for pre-eclampsia (VIP trial): randomised placebo-controlled trial. Lancet 2006;367:1145-1154.

7 Rumbold AR, Crowther CA, Haslam RR, Dekker GA, Robinson JS, ACTS Study Group: Vitamins $\mathrm{C}$ and $\mathrm{E}$ and the risks of preeclampsia and perinatal complications. $\mathrm{N}$ Engl J Med 2006;354:1796-1806.

8 Than NG, Sumegi B, Than GN, Berente Z, Bohn $\mathrm{H}$ : Isolation and sequence analysis of a cDNA encoding human placental tissue protein 13 (PP13), a new lysophospholipase, homologue of human eosinophil Charcot-Ley- den crystal protein. Placenta 1999;20: 703-710.

9 Burger O, Pick E, Zwickel J, Klayman M, Meiri H, Slotky R, Mandel S, Rabinovitch L, Paltieli Y, Admon A, Gonen R: Placental protein 13 (PP-13): effects on cultured trophoblasts, and its detection in human body fluids in normal and pathological pregnancies. Placenta 2004;25:608-622.

10 Nicolaides KH, Bindra R, Turan OM, Chefetz I, Sammar M, Meiri H, Tal J, Cuckle HS A novel approach to first-trimester screening for early pre-eclampsia combining serum PP-13 and Doppler ultrasound. Ultrasound Obstet Gynecol 2006;27:13-17.

11 Spencer K, Cowans NJ, Chefetz I, Tal J, Meiri H: First-trimester maternal serum PP-13, PAPP-A and second-trimester uterine artery Doppler pulsatility index as markers of preeclampsia. Ultrasound Obstet Gynecol 2007; 29:128-134.

12 National Medical Research Council: Clinical Practice Guidelines: Management of Preterm Labour. Singapore, Ministry of Health, 2001, pp 1-22.

13 Brown MA, Lindheimer MD, de Swiet M, van Assche A, Moutquin JM: The classification and diagnosis of the hypertensive disorders of pregnancy: statement from the International Society for the Study of Hypertension in Pregnancy (ISSHP). Hypertens Pregnancy 2001;20:IX-XIV.

14 Davey DA, MacGillivray I: The classification and definition of the hypertensive disorders of pregnancy. Am J Obstet Gynecol 1988; 158:892-898.

15 Knight M, Redman CW, Linton EA, Sargent IL: Shedding of syncytiotrophoblast microvilli into the maternal circulation in preeclamptic pregnancies. Br J Obstet Gynaecol 1998; 105:632-640.
16 Jauniaux E, Watson AL, Hempstock J, Bao YP, Skepper JN, Burton GJ: Onset of maternal arterial blood flow and placental oxidative stress: a possible factor in human early pregnancy failure. Am J Pathol 2000;157: 2111-2122.

17 Mayhew TM, Barker BL: Villous trophoblast: morphometric perspectives on growth, differentiation, turnover and deposition of fibrin-type fibrinoid during gestation. Placenta 2001;22:628-638.

18 Wright DE, Bradbury I: Repeated measures screening for Down's syndrome. Br J Obstet Gynaecol 2005; 12:80-83.

19 Baumann MU, Bersinger NA, Surbek DV: Serum markers for predicting pre-eclampsia. Mol Aspects Med 2007;28:227-244.

20 Papageorghiou AT, Leslie K: Uterine artery Doppler in the prediction of adverse pregnancy outcome. Curr Opin Obstet Gynecol 2007;19:103-109.

21 Allaire AD, Ballenger KA, Wells SR, McMahon MJ, Lessey BA: Placental apoptosis in preeclampsia. Obstet Gynecol 2000;96:271276.

22 Chung EY, Kim SJ, Ma XJ: Regulation of cytokine production during phagocytosis of apoptotic cells. Cell Res 2006;16:154-161.

23 Smith SC, Baker PN, Symonds EM: Increased placental apoptosis in intrauterine growth restriction. Am J Obstet Gynecol 1997;177: 1395-1401.

24 Huppertz B, Kingdom JC: Apoptosis in the trophoblast - role of apoptosis in placental morphogenesis. J Soc Gynecol Investig 2004; 11:353-362.

25 Goswami D, Tannetta DS, Magee LA, Fuchisawa A, Redman CW, Sargent IL, von Dadelszen P: Excess syncytiotrophoblast microparticle shedding is a feature of early-onset pre-eclampsia, but not normotensive intrauterine growth restriction. Placenta 2006;27: 56-61. 